\title{
Eficiência do creep feeding sobre o desempenho de ovelhas Ideal e cordeiros Merino
}

\section{Australiano x Ideal}

\author{
Efficiency of creep feeding on the performance of Ideal sheeps and Merino Australiano $x$ Ideal
}

lambs

Eficiencia del creep feeding sobre el desempeño productivo de ovejas Ideal y cordeiros Merino

Australiano x Ideal

Recebido: 04/02/2021 | Revisado: 07/02/2021 | Aceito: 11/02/2021 | Publicado: 18/02/2021

\author{
Leonardo de Melo Menezes \\ ORCID: https://orcid.org/0000-0001-8536-0803 \\ Universidade Estadual do Rio Grande do Sul, Brasil \\ E-mail: leonardo-menezes@uergs.edu.br \\ Marcus Vinicius Bentancur Fernandes \\ ORCID: https://orcid.org/ 0000-0002-4276-3298 \\ Universidade Estadual do Rio Grande do Sul, Brasil \\ E-mail: mvbf2@hotmail.com \\ Itubiara Maciel da Silva \\ ORCID: https://orcid.org/ 0000-0001-8138-4277 \\ Universidade Estadual do Rio Grande do Sul, Brasil \\ E-mail: tubmaciel@hotmail.com
}

\begin{abstract}
Resumo
Os índices médios de desmame de cordeiros no estado do Rio Grande do Sul giram em torno de 60\%, tornando a atividade pouco competitiva pela baixa escala de produção. Além disso, parte da safra de animais desmamados não chega a terminação em idade jovem (categoria de cordeiro), o que desvaloriza o produto frente ao consumidor. Assim, justifica-se o estudo de técnicas de nutrição animal diferenciada para esta categoria, no intuito de tornar a pecuária ovina mais rentável. Neste sentido, o presente trabalho teve como objetivo avaliar o efeito da suplementação através do sistema creep feeding sobre o desempenho de um sistema de produção de cordeiros Merino Australiano x Ideal, manejados sobre campo nativo. O experimento foi conduzido no município de Santana do Livramento e compreendeu o período de julho a dezembro de 2020. Foram analisadas as variáveis peso final (PF) dos grupos tratamento e controle após o período de suplementação, ganho médio diário (GMD) entre grupos e escore de condição corporal (ECC) das ovelhas após a desmama. Houve efeito do tratamento tanto para machos quanto para fêmeas, para as variáveis peso final e ganho médio diário. O escore de condição corporal das ovelhas não foi influenciado pelo sistema de creep feeding. O sistema de criação de ovinos sob creep feeding promove incremento no peso e na velocidade de crescimento dos cordeiros, apresentado-se como uma alternativa para intensificação da pecuária ovina.
\end{abstract}

Palavras-chave: Carne ovina; Escore de condição corporal; Ganho de peso; Lã; Ovinocultura; Suplementação.

\begin{abstract}
The average weaning rate of lambs in the state of Rio Grande do Sul is around 60\%, making the activity uncompetitive due to the low scale of production. In addition, part of the crop of weaned animals does not reach the end of life at a young age (lamb category), which devalues the product to the consumer. Thus, the study of different animal nutrition techniques for this category is justified, in order to make sheep farming more profitable. In this sense, the present work had as objective to evaluate the effect of the supplementation through the creep feeding system on the performance of a production system of lambs Australian Merino x Ideal, managed on native field. The experiment was conducted in the municipality of Santana do Livramento and covered the period from July to December 2020. The variables final weight $(\mathrm{FW})$ of the treatment and control groups after the period of supplementation, average daily gain (ADG) between groups and body condition score (BSC) of sheep after weaning. There was an effect of the treatment for both males and females, for the variables final weight and average daily gain. The sheep's body condition score was not influenced by the creep feeding system. The system of raising sheep under creep feeding promotes an increase in the weight and growth speed of lambs, presenting itself as an alternative to intensify sheep farming.
\end{abstract}

Keywords: Sheep meat; Body condition score; Weight gain; Wool; Sheep farming; Supplementation. 


\begin{abstract}
Resumen
La tasa promedio de destete de los corderos en el estado de Rio Grande do Sul es de alrededor del 60\%, lo que hace que la actividad no sea competitiva debido a la baja escala de producción. Además, uma parte de animales destetados no llega al final de la vida a una edad temprana (categoría cordero), lo que devalúa el producto para el consumidor. Así, se justifica el estudio de diferentes técnicas de nutrición animal para esta categoría, con el fin de rentabilizar la ganadería ovina. En este sentido, el presente trabajo tuvo como objetivo evaluar el efecto de la suplementación a través del sistema de creep feed sobre el desempeño de un sistema de producción de corderos australianos Merino x Ideal, manejados en campo nativo. El experimento se realizó en el municipio de Santana do Livramento y cubrió el período de julio a diciembre de 2020. Las variables evaluadas fueron el peso final (PF) de los grupos tratamiento y control después del período de suplementación, ganancia diaria promedio (GMD) entre grupos y puntaje de condición corporal (PCC) de las ovejas después del destete. Hubo efecto del tratamiento tanto para machos como para hembras, para las variables peso final y ganancia diaria promedio. La condición corporal de la oveja no fue influenciada por el sistema de alimentación. El sistema de cría de ovinos en alimentación por creep promueve un aumento del peso y velocidad de crecimiento de los corderos, presentándose como una alternativa para intensificar la ganadería ovina.
\end{abstract}

Palabras clave: Carne ovina; Condición corporal; Aumento de peso; Lana; Ganadería ovina; Suplementación.

\title{
1 Introdução
}

A produção de ovinos tem crescido no Brasil, colocando-se como mais uma alternativa à disposição do produtor rural, adaptada aos mais distintos sistemas de produção, desde os mais simples até aos mais tecnificados. Nas últimas décadas, a atividade econômica no Rio Grande do Sul foi voltada para o setor de carne, pela desvalorização do produto lã. Ainda assim, existe expressivo número de produtores voltados ao mercado de lãs finas, estas sim, valorizadas. O consumidor de carne ovina está mais exigente, e no tocante a qualidade exige padronização, desejando uma carne macia e com pouca gordura (Souza, 2018). Esta deve ser proveniente de um animal jovem (categoria cordeiro), com idade máxima ao redor de um ano. Neste sentido, existe necessidade de fornecimento de uma nutrição de qualidade diferenciada para animais que serão abatidos jovens, pela sua alta demanda energética e protéica.

No Rio Grande do Sul, em função do marcado fotoperíodo negativo característico na reprodução das matrizes, o nascimento dos cordeiros ocorre predominantemente nos meses de inverno. No período primaveril-estival, portanto, deve-se priorizar o crescimento e terminação dos cordeiros, visando a obtenção de um produto valorizado e ainda a oferta de dias de descanso produtivo às matrizes, prévios a próxima estação de acasalamento (outono). Seguindo esta linha de raciocínio, nos últimos anos alguns trabalhos e técnicas tem sido centralizadas em alternativas nutricionais para o período estival para engorda e terminação de cordeiros. Estas englobam um espectro muito amplo de manejos e tecnologias, desde as extensivas como acesso restrito à pastagens (Bianchi et al. 2006, Garibotto et al. 2008, De Freitas et al. 2012, Munhoz et al, 2020), utilização de pastagens temporárias (Menezes et al, 2017), suplementação em pastagens (Banchero et al. 2000, Bianchi 2007), creep feeding (Nicola y Saravia 1995, Banchero et al. 2006, Lamarca et al. 2013), até as mais intensivas, como confinamento de cordeiros (Bianchi, 2014; Maydana et al 2019 a; Maydana et al, 2019b). Especificamente sobre o creep feeding, Poli et al. (2008) indicaram que cordeiros mantidos com suas mães em pastagens, com ou sem suplementação em cochos privativos, apresentaram resultados satisfatórios, inclusive quanto à condição sanitária. Neste sentido, Sampaio et al. (2002) relataram que o uso do "creep feeding" pode encurtar o tempo necessário ao acabamento dos cordeiros para o abate, além de proporcionar descanso à matriz. Dentro desse contexto, este trabalho foi realizado com o objetivo de avaliar o desempenho de cordeiros e cordeiras Merino Australiano $\mathrm{x}$ Ideal criados sob sistema creep feeding.

\section{Materiais e Métodos}

O experimento foi conduzido no município de Santana do Livramento (RS), em uma propriedade no $5^{\circ}$ distrito denominada São Diogo, distante $87 \mathrm{~km}$ do centro da cidade. O experimento compreendeu o período de julho a dezembro de 2020. Foram utilizadas ovelhas adultas da raça Ideal, acasaladas previamente com carneiros Merino Australiano. 
O período de parição deu-se entre os dias 25 de julho e 10 de agosto de 2020. Durante este período, os 60 primeiros cordeiros (as) sendo 30 fêmeas e 30 machos foram selecionados ao nascimento para participar do projeto. A seleção dos cordeiros foi realizada através da ordem de nascimento, da seguinte forma: o primeiro a nascer macho foi destinado ao grupo controle; o seguinte, ao grupo tratamento. Da mesma forma realizou-se a seleção das fêmeas. No total, 15 machos e 15 fêmeas foram alocados em cada grupo, totalizando 30 unidades experimentais.

No início do experimento as ovelhas foram avaliadas mensurando-se o escore de condição corporal (ECC). Segundo Machado Fernandes et al. (2008) o ECC é uma medida subjetiva baseada na classificação dos animais em função da cobertura muscular e da massa de gordura, tendo por objetivo estimar o estado nutricional por meio de avaliação visual e/ou tátil, representando uma ferramenta importante de manejo. Neste, atribui-se uma nota que varia de 1 a 5 (muito magra a muito gorda). O método é rápido, prático e barato, reflete as reservas energéticas dos animais e serve como auxiliar na indicação de práticas a serem adotadas no manejo nutricional do rebanho.

No dia 14 de agosto iniciou-se suplementação, através do sistema creep feeding para os cordeiros (as) pertencentes ao grupo tratamento. A ração ofertada continha $18 \%$ de proteína bruta. O consumo diário ofertado foi de $1 \%$ do peso vivo do lote, sendo ajustado a cada 21 dias, através de pesagens realizadas com auxílio de balança digital com capacidade para $500 \mathrm{~kg}$ e precisão de 100 gramas. Os cordeiros do grupo tratamento foram alocados em uma área de 40 hectares próximo da sede da propriedade para otimizar o manejo durante a suplementação, em conjunto mais 10 bovinos sobre a mesma área. Os cordeiros que ficaram no campo nativo foram alocados em um potreiro contíguo, com carga animal equivalente.

Durante o experimento realizou-se uma coleta de pastagem para avaliação quanti-qualitativa da forragem disponível aos animais. Para tanto, utilizou-se um quadro de $1 \mathrm{~m}^{2}$, e o material foi coletado com auxílio de uma tesoura de esquila, utilizada para realização do corte rente ao solo. A coleta foi realizada em dez distintos pontos de cada potreiro aonde estavam alocados os grupos experimentais. O material coletado foi devidamente identificado e mensurou-se o peso das amostras com auxílio de balança digital, registrando-se o peso da matéria verde. O material foi levado a estufa de ventilação forçada de ar (105 C) até peso obtenção de constante e posteriormente calculada a quantidade de matéria seca (MS) por hectare. Após secagem do material e correção do cálculo por hectare obteve-se 4.800 e $3.160 \mathrm{~kg}$ de MS/ha para os grupos tratamento e controle, respectivamente. Após o registro do peso da matéria seca o material foi enviado para o laboratório de nutrição animal da empresa Languiru, localizado em Estrela (RS), para análise bromatológica. Os resultados referentes as análises realizadas podem ser visualizadas na Tabela 1. 
Tabela 1 - Resultados referentes a média das análises bromatológicas para proteína, extrato etéreo, matéria mineral, fibra bruta, FDN e FDA para os potreiros que alocaram os animais de acordo com os grupos tratamento e controle.

\begin{tabular}{ccc}
\hline & Grupo Tratamento & Grupo Controle \\
Proteína (\%) & 8,6 & 11,6 \\
Extrato etéreo (\%) & 1,3 & 1,8 \\
Matéria Mineral (\%) & 13,6 & 11,3 \\
Fibra bruta (\%) & 20,3 & 21,0 \\
FDN (\%) & 53,6 & 55,6 \\
FDA (\%) & 25,6 & 25,0 \\
\hline
\end{tabular}

Fonte: Autores.

O experimento teve duração de 105 dias, quando ao dia 27 de novembro encerrou-se a suplementação. O escore de condição corporal das ovelhas foi aferido novamente neste dia; desta forma, o ECC dos dois grupos foi aferido pelo mesmo técnico em dois momentos: início e término do período de suplementação.

As variáveis consideradas foram o peso ao final do período experimental, ganho médio diário (GMD) dos cordeiros e ECC das ovelhas ao início e fim do experimento. Os efeitos fixos foram o sexo e tratamentos. Os dados foram analisados através de teste de comparação de médias, comparadas pelo teste t para duas amostras. Para tanto, utilizou-se pacote estatístico do Excel.

\section{Resultados e Discussão}

Os resultados obtidos referentes ao desempenho dos cordeiros (as) podem ser visualizados na Tabela 2.

Tabela 2 - Desempenho de cordeiros Merino Australiano x Ideal de acordo com o sexo (macho/fêmea) e tratamento (grupo creep feeding/grupo controle).

\begin{tabular}{ccccc}
\hline & & \multicolumn{3}{c}{ Sexo } \\
\hline Tratamento & Macho & Fêmea \\
Creep Feeding & PF & GMD & PF & GMD \\
Controle & $32,81^{\mathrm{a}}$ & $0,250^{\mathrm{a}}$ & $32,5^{\mathrm{a}}$ & $0,240^{\mathrm{a}}$ \\
\hline
\end{tabular}

$\mathrm{PF}=$ Peso Final GMD = Ganho médio diário. Letras diferentes dentro das colunas representam diferença entre si pelo teste $\mathrm{t}(\mathrm{P}<0,05)$. Fonte: Autores.

Houve efeito do tratamento tanto para machos quanto para fêmeas, para as variáveis peso final e ganho médio diário. Assim, comprovou-se a eficiência da suplementação pelo sistema de cria em creep feeding. Os cordeiros(as) que foram suplementados com o creep feending atingiram peso superior a $31 \mathrm{~kg}$. Tonetto et al. (2004) encontraram rendimento de carcaça quente superior a 52\% em cordeiros alimentados com suas mães, com peso de abate em torno dos $31 \mathrm{~kg}$; os dados também vão de encontro com o s dados obtidos por Figueiró (1989), que descreve que a idade de abate dos cordeiros deve ser entre 90 e 100 dias, com peso vivo de 25 a $30 \mathrm{~kg}$ ao abate. Neste sentido, verifica-se que a implementação da técnica apresenta potencial para gerar carnes e carcaças com excelente padrão de qualidade.

A velocidade de ganho de peso também é um fator importante em cordeiros, e houve diferença entre os tratamentos. Os dados obtidos neste trabalho se aproximaram o encontrado por Carneiro et al. (2004), que obtiveram GMD de 0,260kg utilizando animais do cruzamento entre carneiro Texel e ovelhas mestiças (Texel x Ideal), suplementados com silagem de milho a vontade 
e concentrado na ordem de $0,5 \%$ do peso vivo. Ressalta-se que no referido estudo utilizou-se nível mais intensivo de suplementação (milho ad libitum) e trabalhou-se com animais com maior potencial de ganho de peso, com predominância de raças de corte (Texel). Da mesma forma, Poli et al. (2008) utilizando pastagem de verão (Cynodon spp. cv. Tifton 85) encontrou ganhos semelhantes em cordeiros da raça Suffolk, da ordem de 0,281 kg.

Os ganhos médios diários registrados neste estudo foram inferiores aos encontrados por Neres et al. (2001) e Tonetto et al. (2004), que observaram ganho médio diário de 350 e $325 \mathrm{~g} \mathrm{em}$ cordeiros mantidos em creep feeding do nascimento ao abate. Entretanto, cabe salientar que estes estudos foram realizados com raças de aptidão para carne ou cruzamento com as mesmas; o presente estudo utilizou animais considerados de raças laneiras, o que pode explicar ganhos inferiores.

Os resultados obtidos podem ser visualizados na Tabela 3.

Tabela 3 - Escore de condição corporal (ECC) de ovelhas Ideal no início e fim da suplementação de seus cordeiros (as) em sistema creep feeding.

\begin{tabular}{ccc}
\hline & \multicolumn{2}{c}{ Escore de Condição Corporal (ECC) } \\
\hline Tratamento & ECC inicial & ECC final \\
Creep Feeding & $2,90^{\mathrm{NS}}$ & $3,28^{\mathrm{NS}}$ \\
Controle & $3,10^{\mathrm{NS}}$ & $3,08^{\mathrm{NS}}$ \\
\hline
\end{tabular}

$\mathrm{PF}=$ Peso Final; GMD = Ganho médio diário. Valores de significância obtidos pelo test $\mathrm{t}$, avaliados dentro das colunas. Fontes: Autores .

Não houve diferença entre o ECC de ovelhas que tiveram seus cordeiros suplementados ou não (grupo controle). Rabassa et al (2009) registraram que em sistemas exclusivamente pastoris, baseados em pastagem nativa ocorrem perdas produtivas decorrentes do catabolismo gerado pelo fim de gestação e lactação. Neste sentido, a condição corporal ao fim da lactação e início da estação de monta subsequente são importantes para a obtenção de altos níveis reprodutivos. A maioria dos trabalhos utiliza técnicas como o flushing para aumentar o status energético de ovelhas de cria, melhorando a performance reprodutiva através de índices de gestação, partos gemelares ou mesmo concentração e cios no início do acasalamento (Coop, 1962; Branca et al, 2000; Guedes et al, 2020). Trabalhos que avaliam a condição corporal de ovelhas como variável para tratamento de cordeiros em creep feeding são escassos. Albuquerque et al. (2007), citam que ovelhas com escore corporal maior ou igual a 2,5 apresentam melhor desempenho reprodutivo quando comparadas com animais de condição corporal mais baixa; neste sentido, pode-se verificar que as ovelhas do trabalho em questão apresentam condição corporal satisfatória para o início da próxima estação de acasalamento.

Desta forma, o sistema de creep feeding pode ser uma opção para a manutenção da condição corporal em ovelhas em lactação, além dos ganhos de peso em seus cordeiros. Cabe ressaltar que no presente experimento participaram apenas ovelhas adultas e mães de partos simples; no caso de borregas ou cordeiras (fêmeas jovens) e de partos gemelares o efeito pode ser maximizado, pelo fato de que as demandas energéticas serem maiores nestes casos.

\section{Considerações Finais}

Cordeiros Merino Australiano x Ideal submetidos ao sistema de criação sob creep feeding apresentam desempenho superior quando comparado ao grupo controle. O escore de condição corporal não é influenciado pelo sistema de cria em creep feeding em ovelhas Ideal adultas. Mais variáveis e condições experimentais devem ser estudadas, como tempo de suplementação, categoria utilizada, tamanho de amostra entre outros, em função dos resultados estatísticos obtidos. 
Além disso, variáveis como características de carcaças nos machos e desempenho reprodutivo nas fêmeas podem ser inseridos em estudos mais amplos, para avaliação do impacto da aplicação desta técnica sobre o ganho global do sistema de produção.

\section{Referências}

Albuquerque F. H. M. A. R., Martins G. A., Rogério M. C. P., Memória, H. Q., Sousa, R. T., Simeão R. S. F., Sales, C. M. P. V., Magalhães A. F.B. \& Macedo Júnior G. L. (2007). Efeito da condição corporal antes da estação monta sobre o desempenho produtivo de ovelhas Santa Inês. Anais XVII Congresso Brasileiro de Zootecnia.

Banchero, G., Montossi, F., San Julián, R, Ganzábal, A. \& Ríos, M. (2000). Tecnologías de producción de carne ovina de calidad en sistemas ovinos intensivos del Uruguay. Tacuarembó, INIA. 43 p. (Serie Técnica no. 118).

Bianchi, G. (2007). Alternativas tecnológicas para la producción de carne ovina de calidad en sistemas pastoriles. Buenos Aires, Hemisferio Sur. 283 p.

Bianchi, G. (2014). Confinamiento de corderos. In: Seminario de Actualización Técnica sobre Producción de Carne Ovina de Calidad. Montevideo. Trabajos presentados. Montevideo, INIA. pp. 113-125 (Serie Técnica no. 221).

Bianchi, G., Garibotto, G., Peculio, A., Soca, P., Bentancur, O., Lawlor, F., Ortiz, D. \& Rosales, I. (2006). Efecto del control del tiempo de pastoreo y de la suplementación sobre el desempeño de corderos pesados. In: Congreso Argentino de la Asociación Argentina de Producción Animal. Mar del Plata, Argentina. Trabajos presentados. Revista Argentina de Producción Animal. 26 (supl.1): s.p

Branca, A., Molle, G. \& Sitzia, M. Short-term dietary effects on reproductive wastage after induced ovulation and artificial insemination in primiparous lactating Sarda ewes. Animal Reproduction Science, 58, 59-71, https://doi.org/10.1016/S0378-4320(99)00079-2

Carneiro, R. M., Pires, C. C., Muller, L., Kippert, C. J., Costa, M. L., Colomé, L. M \& Osmari, E. K. Ganho de peso e eficiência alimentar de cordeiros de parto simples e duplo desmamados aos 63 dias e não desmamados. Revista Brasileira de Agrociência. 10(2), 227- 230, (2004). https://doi.org/10.18539/cast.v10i2.948

Coop, I. E. (1962). Effect of flushing on reproductive performance of ewes. Journal of Agricultural Science, 7, $3705-23$.

https://doi.org/10.1017/S0021859600017317

De Freitas, A., Milburn, V. \& Pastorino, V. 2012. Efecto de la suplementación y la restricción estival del tiempo de pastoreo de alfalfa sobre el desempeño productivo de corderos cruza. Tesis Ing. Agr. Montevideo, Uruguay. Facultad Agronomía. 149 p.

Figueiró, P. R. P. (1989). Manejo alimentar do rebanho ovino. In: Simpósio Paulista De Ovinocultura, Campinas. Anais... Campinas: Fundação Cargil, 22-33.

Garibotto, G. \& Bianchi, G. (2008). Alternativas para mejorar la invernada de corderos. Producción Ovina no. 20: 61-76. http://www.produccionanimal.com.ar/produccion_ovina/produccion_ovina_carne/05-ovinos_41-96_alternativas.pdf

Guedes, T. M. F., Maydana, G. M., Santos, R. M. L., Rosa, K. B., Escobar, R. F. \& Menezes, L. M. Efeito do flushing sobre o desempenho reprodutivo de cordeiras Ideal. 2020.In: 35 Jornada Acadêmica Integrada da UFSM - Santa Maria.

Lamarca-Bianchessi, M., Garibotto-Carton, G., Bianchi-Olascoaga, G. \& Bentancourtmurgiondo, O. (2013). Creep feeding en corderos sobre pastizal nativo, efecto del tamaño de camada y del biotipo materno. Abanico Veterinario. 3(2): 22-30. https://dialnet.unirioja.es/servlet/articulo?codigo=7387346

Machado Fernandes, M. A., Gomes Monteiro, A. L., Candal Poli, C. H. E., De Barros, C., Días Ribeiro, T. M. \& Palhano Silva, A. L. (2008). Características das carcaças e componentes do peso vivo de cordeiros terminados em pastagem ou confinamento. Acta Scientarum. Animal Science. 30 (1): 75-81. https://doi.org/10.4025/actascianimsci.v30i1.3617

Maydana, G. M., Santos, R. M. L., Fontoura, E. A. B., Munhoz, M. L., Osorio, T. M., \& Menezes, L. M. (2019ª). Desempenho de cordeiros Merino Dohne em confinamento. In: 19 Congresso Estadual de Medicina Veterinária da SOVERGS, 2019, Gramado.

Maydana, G. M., Santos, R. M. L., Fontoura, E. A. B., Rosa, K. B., Osorio, T. M. \& Menezes, L. M. (2019b). Desempenho de ovinos Corriedale em confinamento. In: 11 Salão Internacional de Pesquisa, Ensino e Extensão, Santana do Livramento/Rivera.

Nicola, A. L., Saravia, C. G. (1995). Efecto de la suplementación a corderos al pie de sus madres - (Creep feeding). Tesis Ing. Agr. Montevideo, Uruguay. Facultad de Agronomía.

Menezes, L. M., Fontoura, E. A. B., Damilano, A. S., Rosa, R. S., Perez, H. A., Gomes, A. F. F., Cunha, P. T., Chagas, R. A. \& Corrêa, G. F. (2017). Desempenho de cordeiros Texel e Corriedale mantidos em azevém em fím de ciclo. Revista electrónica de Veterinaria. 18(12):1-9. https://www.redalyc.org/pdf/636/63654640033.pdf

Munhoz, M. L., Fontoura, E. A. B., Rodrigues, D. P., Moreira, C. H., Rodrigues, P. E. B., Cordeiro, D. O., Santana, G. A. O. \& Menezes, L. M. (2020). Desempenho de ovelhas e cordeiros Texel em distintas fases do manejo nutricional. Brazilian Journal of Development, 6, $4909-4919$. https://doi.org/10.34117/bjdv6n1-353

Neres, M. A., Monteiro, A. L. G., Garcia, C. A., Costa, C., Arrigoni, M. B. \& Rosa, G. J. M. 2001. Forma física da ração e pesos deabate nas características de carcaça de cordeiros em creep feeding. Revista Brasileira de Zootecnia, 30(3), 948-954, 2001. https://doi.org/10.1590/S1516-35982001000400007

Poli, C. H. E. C., Monteiro, A. L. G., Barros, C. S., Moraes, A., Machado Ferreira, M. A. \& Piazzeto, H. V. L. Produção de ovinos de corte em quatro sistemas de produção. Revista Brasileira de Zootecnia, 37(4), 666-673. https://doi.org/10.1590/S1516-35982008000400012 
Research, Society and Development, v. 10, n. 2, e34110212663, 2021

(CC BY 4.0) | ISSN 2525-3409 | DOI: http://dx.doi.org/10.33448/rsd-v10i2.12663

Rabassa, V. R., Tabeleão, V. C., Schneider, A., Menezes, L. M., Schossler, E., Severo, N., Schwegler, E., Goulart, M. A., Del Pino, F. A. B., Nogueira, C. E. W. \& Corrêa, M. N. 2009. Avaliação metabólica de ovelhas de cria mantidas em campo nativo durante o período de outono/inverno. Revista Brasileira de Agrociência, 15(1-4), 125-128. https://doi.org/10.18539/cast.v15i1-4.1999

Sampaio, A. A. M., Brito, R. M. \& Routman, K. S. Utilização de NaCl no suplemento com alternativa de viabilizar o creep feeding. 2001.In: Reunião Anual Da Sociedade Brasileira De Zootecnia, 2001, Piracicaba. Anais...Piracicaba: Sociedade Brasileira de Zootecnia, 2001. 987-988.

Souza, T. H. Levantamento de características fenotípicas de abate em ovinos de diferentes origens e tipos biológicos comercializado na região metropolitana de Porto Alegre - RS. 2018. 61 f. Monografia (graduação) - Universidade Federal do Rio Grande do Sul - Curso de Zootecnia, Porto Alegre.

Tonetto, C. J., Pires, C. C., Müller, L., Rocha, M. G., Silva, J. H. S., Cardoso, A. R. \& Peres Neto, D. (2004). Ganho de peso e características da carcaça de cordeiros terminados em pastagem natural suplementada, pastagem cultivada de azevém (Lolium multiflorum Lam.) e confinamento. Revista Brasileira de Zootecnia, 33(1), 225-233. http://dx.doi.org/10.1590/S1516-35982004000100026 